\title{
Measurements of Available Bandwidth in Computer Networks
}

\author{
Kire Jakimoski ${ }^{1}$, Sime Arsenovski ${ }^{2}$, Lidija Gorachinova $^{3}$, Slavcho Chungurski ${ }^{4}$ \\ Oliver Iliev $^{5}$, Leonid Djinevski ${ }^{6}$, Emilija Kamcheva ${ }^{7}$ \\ 1,2,3,4,5,6,7 Faculty of Informatics, FON University, Skopje, Republic of Macedonia \\ ${ }^{1}$ kire.jakimoski@fon.edu.mk; ${ }^{2}$ sime.arsenovski@fon.edu.mk; \\ ${ }^{3}$ lidija.goracinova@fon.edu.mk; ${ }^{4}$ slavcho.chungurski@fon.edu.mk; \\ ${ }^{5}$ oliver.iliev@fon.edu.mk; ${ }^{6}$ leonid.djinevski@fon.edu.mk; \\ 'emilija.kamceva@fon.edu.mk
}

\begin{abstract}
Available bandwidth is one the key challenges that is present in every new developed standard in the computer networks. There are many proposed methodologies in the existing research literature for measuring the available bandwidth. In this paper we will present summary of the main contributions in this field, research problems and solutions and we will present possible ways for improving the suggested solutions in the existing literature. Furthermore, survey of the most important tools for measuring the available bandwidth in wired and wireless networks is presented in details.
\end{abstract}

Keywords: available bandwidth, measurement, tools, performances, wired, wireless

\section{Introduction}

There are two general kinds of measurement techniques, active and passive. There are a lot of applications that could benefit from assessments made by active or passive measurement methods. Available bandwidth is one of the key network characteristics and the usage of passive and active measurement methods are usual strategies to measure it.

The available bandwidth is deeply researched and a lot of measurement algorithms are proposed in the existing research papers. That is why in this work we examine the main research problems and solutions.

The available bandwidth of a single link can be defined as:

$A_{i}=\left(1-u_{i}\right) * C_{i}$

where $A_{i}$ is the available bandwidth on link $i, u_{i}$ is the utilization of link $i$, and $C_{i}$ is the capacity of link $i$. Furthermore, end-to-end available bandwidth of a path that contains $H$ links is defined as the minimum available bandwidth:

$$
A=\min _{i=1 \ldots H} A_{i}
$$

One of the main challenges in the research of the bandwidth measurement is the fact that the available bandwidth is varying as time passes. This is result of the inconsistency of the cross traffic over time. Cross traffic presents the normal traffic that is transmitted over the network, not including the probe traffic that is used for measurement purposes.

Another research problem is the interaction between the probe traffic (used for measurement purposes) and the cross traffic on a network link. This challenge needs to be examined from two different aspects, from the probe traffic aspect and from the cross traffic aspect. So, the influence of the cross traffic to probe traffic, as well as the influence of the probe traffic to cross traffic is very interesting research problem. 
There are always novel network technologies that are standardized like $802.16 \mathrm{~m}, 4 \mathrm{G}$, 5G, LTE-Advanced, etc. So, research challenge here is to examine if the present bandwidth measurement techniques are relevant for the new standards of the network technologies or some adjustments are needed.

Serious research problem is how to evaluate and compare the accessible tools for bandwidth measurement. It is not simple to find general criteria that will be objective for comparison of the tools and techniques for bandwidth measurements.

Optimization between the quantity of the probe traffic that is transmitted between the probe sender and the probe receiver and the exactness of the measurement technique is also another research problem in the bandwidth measurements. It is good to have lower quantity of the probe traffic, because the probe traffic is bad for other traffic flows. On the other side, if we decrease the quantity of the probe traffic, we decrease also the exactness of the bandwidth measurements. That is why the optimization is needed between the quantity of the probe traffic and the exactness of the bandwidth measurement technique.

In this work we will present the main methodologies and contributions for measuring the available bandwidth in computer networks. Then we will analyze in more details the most important solutions for measuring the available bandwidth in wired and wireless networks.

\section{Main Methodologies for Measuring the Available Bandwidth}

There are many solutions in the existing research literature for measuring the available bandwidth. A lot of tools for bandwidth measurement are developed and used for measuring the available bandwidth. The most important methods for measuring the available bandwidth are packet rate and packet gap method [1]. Solutions for measuring the available bandwidth using the packet rate method are obtained with the tools Pathload [2], Pathchirp [3], Bart [4], and TOPP [5].

Pathload [2] as a tool for measuring bandwidth is using the SLoPS (Self-Loading Periodic Streams) [6]. SLoPS methodology is measuring end-to-end available bandwidth. Packet train is transmitted by the sender to the receiver with a probe rate that is predefined. The send time is stamped by the sender and the probe packet reception is stamped by the receiver. So, the difference it the time stamp is defined as one way delay OWD and it is computed for every packet. If the initial rate has higher value than the available bandwidth, the queue of the router will raise when supplementary probe packets are received by the router. As a result of this, the values for OWD will grow. But, if the initial rate has lower value than the available bandwidth, the queue of the router won't raise. So, in this case the OWD will be steady. According the above said facts, we set the probe-train rate according the results of the OWD. If OWD is increased by the probe rate, then in the next train we will decrease the probe rate, because it probe rate is higher than the available bandwidth. But, if OWD is not increased, the probe rate is lower than the available bandwidth, so we can increase the probe rate now. This iteration process is repeated till the moment we are assured with the correctness of the obtained available bandwidth.

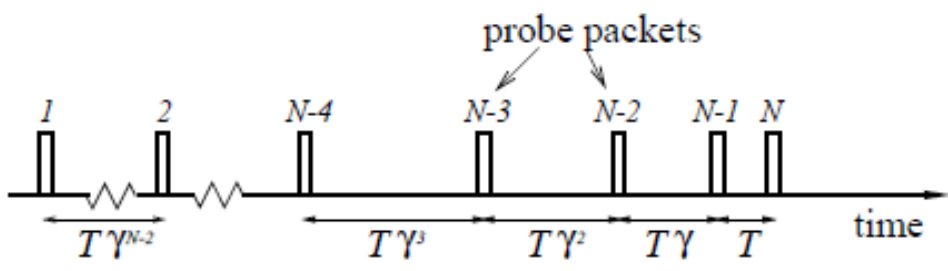

Figure 1. Chirp Probe Train [3] 
Pathchirp [3] is another solution for measuring the end-to-end available bandwidth using the packet rate methodology. Comparing to Pathload, this solution uses 'chirps' of probe packets for sending instead of probe packet trains. Figure. 1 presents the chirp probing train which is exponentially spaced in [3]. Chirps are more efficient than packet pairs because they assess the available bandwidth with using few packets.

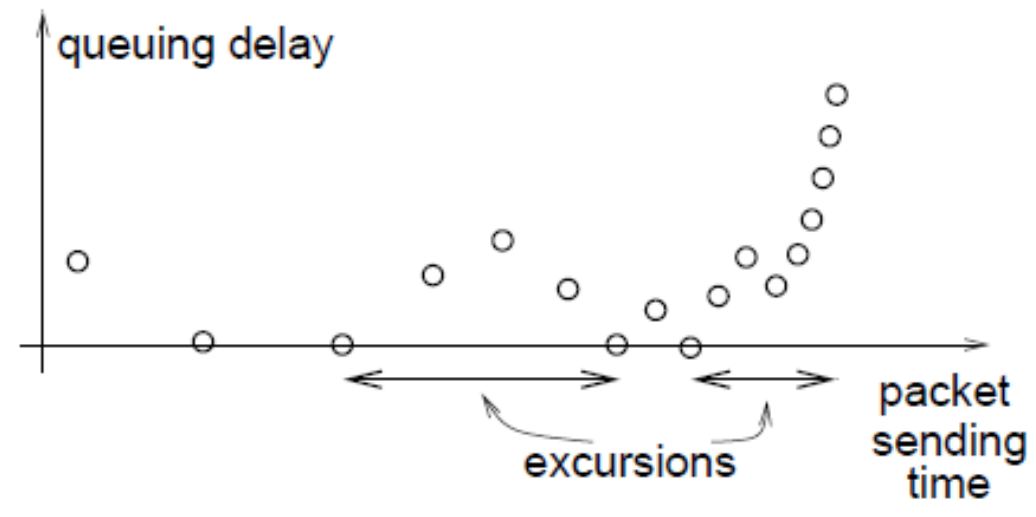

\section{Figure 2. Queuing Delay for a Given Packet Sending Time using the Pathchirp [3]}

Figure. 2 presents the queuing delays of a usual chirp train proposed in [3]. Queuing delay is increasing its value when the initial rate is over the available bandwidth. In Figure. 2 the results are measured using just one chirp. In this part is concluded that when we have excursions the cross traffic is more intensive and it produces queuing. It is important to know that when excursions are getting back to zero the cross traffic is bursty, but when the excursion is not returning to zero (like the last excursion in Figure. 2), that means that the send probe rate is above the allowed capacity of the link. Patchirp methodology uses the excursions from numerous chirps to assess the end-to-end available bandwidth.

The other group of solutions for measuring the available bandwidth is using the packet gap methodology. When this method is used, typically the bottleneck link must be identified. Delphi [7] and Spruce [8] are tools for measuring the available bandwidth based on the packet gap method. Estimation procedure in [7] is model-based, and that is why the proposed Delphi algorithm could adopt an effective probing pattern. So, Delphi uses probing strategy to dynamically assess the cross-trafic load all along the end-to-end network path. This tool for measuring bandwidth is fully adaptive without need for a priori traffic statistics. In [7] the term 'virtual cross-traffic' is used for an end-to-end path. Virtual cross-traffic stands for the aggregated cross-traffic that has to be placed in the single queue model so that the dynamic available bandwidth that is measured is the same as that along the entire multi-hop path. The connection between the dynamic available bandwidth and the virtual cross-traffic is presented in Figure. 3. Authors in [7] verify the proposed algorithm by experiments that are done using the network simulator (ns). The advantage of this tool is that it gives precise cross-traffic measurements when the utilization level is higher. Disadvantage of the Delphi in [7] is that it over-estimates the cross-traffic when the utilization is lower. 


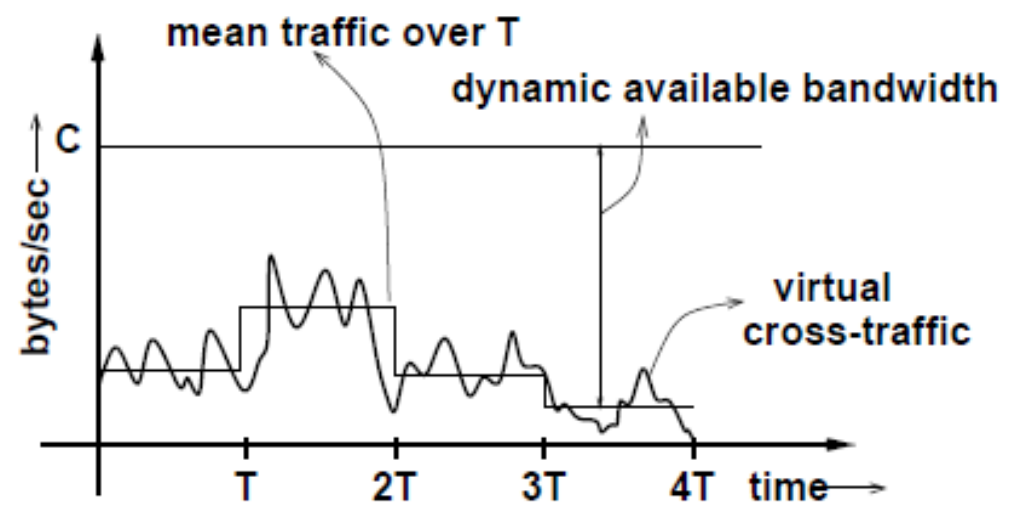

Figure 3. Dynamic Available Bandwidth and Virtual Cross-Traffic [7]

Spruce [8] is light-weight and simple tools that measures the available bandwidth comparing it with two other tools, Pathload and IGI over 400 various Internet paths. Comparative analysis between them is focused on probe overhead, failure patterns, accuracy, and implementation issues. Authors in [8] verify the measuring of the available bandwidth by comparison to MRTG (Multi-Router Traffic Grapher) data. Actually, the response of each tool to induced changes in the available bandwidth is measured here. According to the presented measurement in [8], it gives more precise results than IGI and Pathload tools. In fact, Pathload tool have a propensity to over assess the available bandwidth, while IGI is not sensitive to bottleneck utilization.

\section{Main Contributions for Measurements of Available Bandwidth}

A lot of software tools for measuring the available bandwidth in the networks have been developed in the last 15 years by the researchers and scientists. Various principles and techniques are used for development of these software tools for measuring the available bandwidth. Main contributions in this field are presented in this section.

Available bandwidth as a subject of research is key challenge in the history of computer networks. Regarding the transport protocols, like TCP (Transport Control Protocol), available bandwidth is one of the most important concerns [9]. Estimation of the available bandwidth is extremely valuable for route selection in overlay networks, traffic engineering, and QoS (Quality of Service) verification. Available bandwidth is key metric for illustrating the presenting the performance of a network path. As a result of this, available bandwidth is used in numerous applications.

The most simple and efficient way for assessing the available bandwidth is active probing. This can be done with a few test packets that are sent throughout the network and used to conclude the status of the network. In the past years a lot of active probing tools were developed. Still, providing reliable assessment of the available bandwidth is continuing to be challenging task. The most important conditions for successful measurement of the available bandwidth are correctness, robustness, and non-intrusion at the same time.

In [6] the authors present end-to-end available bandwidth measurement methodology, names as "Self-Loading Periodic Streams or SLoPS". Furthermore, measurement tool named "pathload" [2] is used in [6] and this tool is confirmed experimentally.

In [10] the authors propose effective and practical method to assess the end-to-end available bandwidth, as well as the speed of the most congested link in supposed Internet path. In [10] the main idea is to estimate the end-to-end path utilization throughout a simple binary test. With this test the fraction of probe packets that experienced queuing is measured. Actually, the path utilization is measured on two transmitted probing streams 
with different rates, projecting in this way the available bandwidth and the speed of the congested link.

Novel active probing methodology named COPP (chirp of packet paris) is proposed in [11]. It combines self-induced congested principle with packet pair technique. Authors in this work provide simulation results in several cross traffic conditions and these results point out that COPP is offering accurate assessment of the available bandwidth with relatively small number of overhead compared to other tools.

Authors in [12] present novel approach for estimation of the available bandwidth using stratified probing on access links. Approach in this work is divided in four phases. Firstly, a traffic profile is established for the cross-traffic with focus on the characteristics of the burst and idle sub-periods. Then, the active probing is divided into strata in the second and third phase, and it is synchronized relating to the burst/idle sub periods. In the last phase the probing and estimation of the available bandwidth occurs. Adaptive video streaming in a controlled lab environment is used for experimental analysis of the proposed method for measurement of the available bandwidth. Stratified approach in [12] gives accurate empirical results and low degree of intrusiveness. Still, one of the disadvantages that we can notice in this work is that periodic behavior in the cross-traffic is needed when using this method for measuring the available bandwidth.

\section{Tools for Measuring the Available Bandwidth in Wired Networks}

In the previous sections we have presented methods, tools and models and main contributions for measuring the available bandwidth that are developed in the past 15 years. If we take a look at the big image, all this models, tools and systems are measuring the available bandwidth of some network path by sending several packets and analyzing the outcomes on the probe packets and cross-traffic.

The most important tools for measurement of the available bandwidth that were developed in the wired networks in the last 15 years are Pathload [2], IGI [13], Delphi [7], Spruce [8], Pathchirp [3]. These methods and tools are differing in the temporal structure and the size of probe packets, as well as in the way the available bandwidth is obtained form the received packets.

Pathload in [2] is using constant bit-rate streams and each round the sending rate is changed. Binary search scheme is used from Pathload to vary the probing rate. IGI [13] uses a sequence of around 60 unevenly spaced packets. Network is probed with these packets and the gap between two successive packets is raised until the average output and initial gaps match. When Spruce [8] tool is used, tens of packet pairs are used with an input rate approximately similar to the path capacity. These packets are spaced exponentially in order to have a poissonian sampling process. Pathchirp [3] is sending variable bit-rate stream that includes packets that are spaced exponentially. So, the actual unused capacity is deduced from the rate liable for increasing delays at the receiver.

The above presented methods and tools for measuring the available bandwidth are difficult for thorough and objective comparison. If only one part of the existing tools for measuring available bandwidth is chosen, then the accuracy of the comparative analysis would be suspicious. Furthermore, it is very difficult to compare the results of the experiments if they are performed using various testbed configurations and scenarios.

One of the solutions is to have unified and flexible platform for independent evaluations of the tools for measuring available bandwidth. This kind of solution is given in [14] where testbed solution based on free GPL-licensed software is proposed. In this work 9 software programs are examined in terms of time, accuracy and intrusiveness. So, very large comparison of the tools for measuring available bandwidth is done. According to the results for accuracy, Spruce offers good estimations when the cross-traffic is low, but as the network load is increasing the accuracy decreases significantly. Contrary, if IGI is used the accuracy of measurements is increased when the cross-traffic is higher. 
Pathchirp according to the results obtained in [14], constantly over assesses the available bandwidth and its measurements are unbalanced. Pathload is quite accurate according to the experimental results in [14]. According to the results obtained in [14], IGI is quite fast, but with considerable amount of probe traffic. Spruce, on the other side, is also very fast but it is more lightweight. Pathload is slow and intrusive, but Pathchirp has good trade-off between intrusiveness and speed.

Anyway, weakness of the developed method for comparison of the tools for measurement of available bandwidth in [14] is that longterm oscillations in the assessment or biases are not included. Highly congested scenarios are also not included in the presented analysis. The laboratory testbed that is used in [14] is quite simple, so it doesn't present the complexity of the real communication flows. So, the results obtained from the experiments in [14] are not enough to have relevant conclusions on how the tools will measure the available bandwidth on real networks.

\section{Tools for Measuring the Available Bandwidth in Wireless Networks}

Measurement of the available bandwidth in wireless networks is even more challenging task, because the wireless conditions are unpredictable and unstable. Available bandwidth in this type of networks is also varying because of the shared-access type of the wireless channel, so the number of hosts in the network influences on the available bandwidth. In the existing literature we can find the following methods for measuring the available bandwidth of the wireless networks:

- Probing methodology;

- Cross layer-based methodology;

- $\quad$ Model-based methodology.

Probing methodology requires considerable bandwidth resources, while cross layerbased methodology is connected with modifications of protocols. Model-based methodology is newer technique and it is related to the recently suggested algorithms for getting robust results of the bandwidth measurements.

Probing methodology for measuring the available bandwidth of wireless networks is implemented with the DietTOPP [15], Wbest [16], ProbeGap [17] and AdhocProbe [18].

DietTOPP in [15] is using probing packet pair and the available bandwidth is measured when the probing traffic bit rate is experiencing the turning point. Wbest estimates the effective capacity with probing packet-pair dispersion in the cases where the last hop is WLAN (wireless LAN). Packet-train methodology is employed to assess the achievable throughput in order to deduce the available bandwidth.

The benefit of Wbest in [16] is that it avoids a search algorithm for detection of the available bandwidth and statistically detects the available portion of the effective capacity. In this way the assessment delay is decreased and the influence of random wireless channel errors is also decreased.

ProbeGap solution for measuring the available bandwidth of wireless network probes for gaps in the busy periods and the assessment of the available bandwidth is get by multiplying them by the capacity. Anyway, one of the key disadvantage of the ProbeGap in [17] is that it is dependent on other schemes for capacity assessment.

AdhocProble solution in [18] functions with sending from the sender to the receiver fixed size and back-to-back probing packet pairs. Each of the packets has stamp of the transmission time by the sender. Calculation of the path capacity is then done at the receiver side. One of the main disadvantages of this solution is that it is adequate for calculating the path capacity in wireless networks with fixed rate.

Cross layer based methodology for measuring the available bandwidth in wireless networks is implemented with solutions like IdleGap [19] and iBE [20]. 
IdleGap in [19] uses an idle module between the network and the link layer and link idle rate is obtained by this module from the network allocation vector. This link idle rate is then send to the application layer. So, the available bandwidth with this solution is computed with the known capacity and the link idle rate.

iBE in [20] assesses the available bandwidth of the wireless network with packet dispersion methodology. Using this techique the packet payload size is recorded as well as the OWD (one-way delay) at the MAC layer. After this the assessed results are transmitted to the application layer for intelligent adaptation. One of the advantages of this solution is that the estimation overhead is reduced because instead of using probing traffic, the application data packets are used for that purpose. But, disadvantage of this solution is that it necessitates adjustments of the MAC protocol in 802.11 standard.

If we summarize about the probing-based methodology results, we can conclude that this methodlogy is based on probing traffic and it influences on the services of the wireless communications because of the supplementary involved data.

On the other hand, cross-layer methodology gives lower overhead comparing to packet dispersion results. But, there are complications in the deploying process because of the adjustments that are necessary in the standard protocols and devices.

The probing-based and cross-layer methodologies both require considerable bandwidth resources or adjustments of the protocol in order to measure the available bandwidth of the wireless networks. That is why model-based methodology is recently proposed in order to solve the previous commented challenges of the other two methodologies. Algorithm for assessing the available bandwidth based on this newer technique is suggested in [21]. In [21] the authors propose MBE (Model-based Bandwidth Estimation) algorithm for 802.11 wireless networks for multimedia services. This algorithm is based on novel TCP/UDP throughput models for wireless networks. The advantage of this methodology is that probing traffic is not included and there are no adjustments and modifications of the MAC (Media Access Control) protocol.

Table 1. Summary of Some of the Tools for Measuring Available Bandwidth in Wired and Wireless Networks

\begin{tabular}{|c|c|c|c|}
\hline $\begin{array}{l}\text { Tool for measuring } \\
\text { available bandwidth }\end{array}$ & $\begin{array}{l}\text { Type of network } \\
\text { (wired or } \\
\text { wireless) }\end{array}$ & Measurement metric & Methodology \\
\hline Pathload & Wired & $\begin{array}{l}\text { End-to-end available } \\
\text { bandwidth }\end{array}$ & $\begin{array}{l}\text { Self-loading } \\
\text { periodic streams }\end{array}$ \\
\hline Pathchirp & Wired & $\begin{array}{l}\text { End-to-end available } \\
\text { bandwidth }\end{array}$ & $\begin{array}{l}\text { Self-loading } \\
\text { packet chirps }\end{array}$ \\
\hline$|G|$ & Wired & $\begin{array}{l}\text { End-to-end available } \\
\text { bandwidth }\end{array}$ & $\begin{array}{l}\text { Self-loading } \\
\text { periodic streams }\end{array}$ \\
\hline DietTOPP & Wireless & $\begin{array}{l}\text { Per-hop capacity and } \\
\text { bandwidth }\end{array}$ & $\begin{array}{l}\text { Packet pairs and } \\
\text { trains }\end{array}$ \\
\hline Wbest & Wireless & $\begin{array}{l}\text { Per-hop capacity and } \\
\text { bandwidth }\end{array}$ & $\begin{array}{l}\text { Packet pairs and } \\
\text { trains }\end{array}$ \\
\hline ProbeGap & Wireless & $\begin{array}{l}\text { Path capacity and } \\
\text { bandwidth }\end{array}$ & $\begin{array}{l}\text { Packet pairs and } \\
\text { trains }\end{array}$ \\
\hline AdhocProbe & Wireless & $\begin{array}{l}\text { Path capacity and } \\
\text { bandwidth }\end{array}$ & $\begin{array}{l}\text { Packet pairs and } \\
\text { trains }\end{array}$ \\
\hline IdleGap & Wireless & $\begin{array}{l}\text { End-to-end capacity } \\
\text { and bandwidth }\end{array}$ & Link idle rate \\
\hline iBE & Wireless & $\begin{array}{l}\text { End-to-end capacity } \\
\text { and bandwidth }\end{array}$ & Packet dispersion \\
\hline MBE & Wireless & $\begin{array}{l}\text { End-to-end capacity } \\
\text { and bandwidth }\end{array}$ & $\begin{array}{l}\text { Model based } \\
\text { algorithm }\end{array}$ \\
\hline
\end{tabular}


Table 1 presents the most important solutions for measuring available bandwidth and capacity in wired and wireless networks. It classifies the tools that we examined in details according the type of network, measurement metric and methodology.

\section{Conclusion}

In this work we presented a survey on measurements of the available bandwidth in the computer networks. Motivation, major research problems and solutions in the field of measuring the available bandwidth are presented in details. We also discussed about the main contributions of the listed papers in this field of scientific researches. We also identified open research problems, advantages and disadvantages of the work done in the presented papers. We also discussed about the problems that arise in the implementation and experimentation during the measurements of the available bandwidth in wired and wireless networks.

We can conclude that this subject of research is challenging in the last 15 years and it is still in trend. There are a lot of proposed solutions for measuring the available bandwidth and we reviewed the most important of them. We analyzed advantages and disadvantages of them using comparative analysis and proposed solutions for improving them in future.

\section{References}

[1] Strauss, Jacob, Dina Katabi, and Frans Kaashoek. "A measurement study of available bandwidth estimation tools." Proceedings of the 3rd ACM SIGCOMM conference on Internet measurement. ACM, 2003.

[2] Jain, Manish, and Constantinos Dovrolis. "Pathload: A measurement tool for end-to-end available bandwidth." In Proceedings of Passive and Active Measurements (PAM) Workshop. 2002.

[3] Ribeiro, Vinay J., et al. "pathchirp: Efficient available bandwidth estimation for network paths." Presented at. No. SLAC-PUB-9732. 2003.

[4] Ekelin, Svante, and Martin Nilsson. "Continuous monitoring of available bandwidth over a network path." Proc. 2nd Swedish National Computer Networking Workshop (SNCNW 2004). 2004.

[5] Melander, Bob, Mats Björkman, and Per Gunningberg. "A new end-to-end probing and analysis method for estimating bandwidth bottlenecks." Global Telecommunications Conference, 2000. GLOBECOM'00. IEEE. Vol. 1. IEEE, 2000.

[6] Jain, Manish, and Constantinos Dovrolis. End-to-end available bandwidth: Measurement methodology, dynamics, and relation with TCP throughput. Vol. 32. No. 4. ACM, 2002.

[7] Ribeiro, Vinay, et al. "Multifractal cross-traffic estimation." Proc. of ITC specialist seminar on IP traffic Measurement. 2000.

[8] Strauss, Jacob, Dina Katabi, and Frans Kaashoek. "A measurement study of available bandwidth estimation tools." Proceedings of the 3rd ACM SIGCOMM conference on Internet measurement. ACM, 2003.

[9] Jacobson, Van. "Congestion avoidance and control." ACM SIGCOMM computer communication review. Vol. 18. No. 4. ACM, 1988.

[10] Neginhal, Mradula, Khaled Harfoush, and Harry Perros. "Measuring bandwidth signatures of network paths." NETWORKING 2007. Ad Hoc and Sensor Networks, Wireless Networks, Next Generation Internet. Springer Berlin Heidelberg, 2007. 1072-1083.

[11] LU, Shao-he, et al. "Efficient available bandwidth estimation for network paths."Journal of Harbin Institute of Technology 1 (2008): 032.

[12] Villa, Bjørn Jonny, and Poul Einar Heegaard. "Estimating available bandwidth on access links by means of stratified probing." International Journal of Electrical Energy. Vol. 1. No. 4. ETPublishing, 2013.

[13] $\mathrm{Hu}$, Ningning, and Peter Steenkiste. "Evaluation and characterization of available bandwidth probing techniques." Selected Areas in Communications, IEEE Journal on 21.6 (2003): 879-894.

[14] Goldoni, Emanuele, and Marco Schivi. "End-to-end available bandwidth estimation tools, an experimental comparison." Traffic Monitoring and Analysis. Springer Berlin Heidelberg, 2010. 171182.

[15] Johnsson, Andreas, Bob Melander, and Mats Björkman. "Diettopp: A first implementation and evaluation of a simplified bandwidth measurement method."Second Swedish National Computer Networking Workshop. Vol. 5. 2004.

[16] Li, Mingzhe, Mark Claypool, and Robert Kinicki. "WBest: A bandwidth estimation tool for IEEE 802.11 wireless networks." Local Computer Networks, 2008. LCN 2008. 33rd IEEE Conference on. IEEE, 2008. 
[17] Lakshminarayanan, Karthik, Venkata N. Padmanabhan, and Jitendra Padhye. "Bandwidth estimation in broadband access networks." Proceedings of the 4th ACM SIGCOMM conference on Internet measurement. ACM, 2004.

[18] Sun, Tony, et al. "A measurement study of path capacity in $802.11 \mathrm{~b}$ based wireless networks." Papers presented at the 2005 workshop on Wireless traffic measurements and modeling. USENIX Association, 2005.

[19] Lee, Heung $\mathrm{Ki}$, et al. "Bandwidth estimation in wireless lans for multimedia streaming services." Advances in Multimedia 2007 (2007).

[20] Yuan, Zhenhui, Hrishikesh Venkataraman, and Gabriel-Miro Muntean. "iBE: A novel bandwidth estimation algorithm for multimedia services over IEEE 802.11 wireless networks." Wired-Wireless Multimedia Networks and Services Management. Springer Berlin Heidelberg, 2009. 69-80.

[21] Yuan, Zhenhui, Hrishikesh Venkataraman, and Gabriel-Miro Muntean. "MBE: Model-based available bandwidth estimation for IEEE 802.11 data communications." Vehicular Technology, IEEE Transactions on 61.5 (2012): 2158-2171.

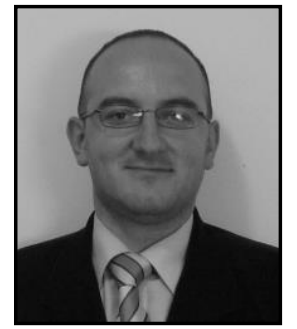

\section{Authors}

Kire Jakimoski. Kire Jakimoski received his B.Sc. degree in the field of Telecommunications from the Military Academy "Mihailo Apostolski" in Skopje, R. Macedonia in 2002, M.Sc. degree in Electrical Engineering in the field of Telecommunications from the Ss. Cyril and Methodius University in Skopje, R. Macedonia in 2007, and $\mathrm{Ph} . \mathrm{D}$. in technical sciences from the Ss. Cyril and Methodius University in Skopje, R. Macedonia in 2013. From 2002 to 2006 he works as an Officer for Telecommunications in the Ministry of Defense in the Republic of Macedonia. From January, 2006 to March, 2012 he works as an adviser for information security in the Directorate for Security of Classified Information in the Republic of Macedonia. From March, 2012 he is with the Faculty of Informatics, FON University in Skopje. Also, he is an author/co-author of above 30 published research papers and one book. He is an Assistant Professor and Vice Dean at the Faculty of Informatics, FON University in Skopje, Macedonia. His research interests include Wireless and Mobile Networks, Heterogeneous Wireless Networks, Computer Networks, Digital Telecommunications, Information Security.

Sime Arsenovski is a Full Professor at the Faculty of Informatics, and Rector of the FON University, Skopje, Republic of Macedonia.

Lidija Gorachinova is a Full Professor at the Faculty of Informatics, and Vice Rector of the FON University, Skopje, Republic of Macedonia.

Slavcho Chungurski is an Associate Professor and Dean at the Faculty of Informatics, FON University, Skopje, Republic of Macedonia.

Oliver Iliev is a Full Professor at the Faculty of Informatics, FON University, Skopje, Republic of Macedonia.

Leonid Djinevski is an Assistant Professor at the Faculty of Informatics, FON University, Skopje, Republic of Macedonia.

Emilija Kamcheva is an Assistant Professor at the Faculty of Informatics, FON University, Skopje, Republic of Macedonia. 
International Journal of Grid and Distributed Computing Vol. 9, No. 4 (2016) 